\title{
Benefits of Cholesterol Screening and Therapy for Primary Prevention of Cardiovascular Disease: A New Paradigm
}

One third of the approximately 1.5 million Americans who will experience a myocardial infarction (MI) in the United States this year will die; survivors will have serious morbidity, including congestive heart failure, arrhythmias, and an increased risk of sudden cardiac death. ${ }^{1}$ Thus, the prevention of coronary heart disease (CHD), before its clinical manifestations, is an important public health issue. Cholesterol screening is firmly established as an effective strategy for CHD prevention because lipid-lowering therapy for patients with hypercholesterolemia reduces cardiac morbidity and mortality. ${ }^{2-6}$

In this issue of the fournal, Froom et al argue that lipid-lowering therapy for the primary prevention of cardiovascular events is unwarranted "with the possible exception of men aged 35 to 59 " years. ${ }^{7}$ They criticize the National Cholesterol Education Program (NCEP) guidelines, especially in regard to primary prevention recommendations for women, young men, and elderly patients. 2,7 They reached their conclusions by (1) an admittedly selective review of data from five major primary prevention trials, (2) a misleading interpretation of the results of the West of Scotland Coronary Prevention Study (WOSCOPS), and (3) reliance on selected observational studies of cholesterol levels and CHD risk in women and older patients. ${ }^{7}$

It is crucial to clarify some of the misrepresentations of Froom et al regarding cholesterol screening and lipid-lowering therapy in patients without CHD. We maintain that a scientifically rigorous overview of the results of all the primary prevention trials that takes into consideration the

Submitted 25 August 1997.

From the Preventive Cardiology Program, Section of Cardiology, Departments of Medicine (JHS, PEM) and Family Medicine (PEM), University of Wisconsin Medical School, Madison. Address reprint requests to Patrick E. McBride, MD, MPH, University of Wisconsin Medical School, 600 Highland Ave, H6/349 CSC, Madison, WI 53792. results of several recent, prospective, randomized, clinical trials renders criticisms of the older studies irrelevant. $4,8,9$ Many of the trials cited by Froom et al studied lipid-lowering therapies that are no longer used except in life-threatening situations (clofibrate) or are unavailable for clinical use (probucol). More recent clinical trials, which used more potent and safer agents, validated the rationale behind the NCEP recommendations for cholesterol screening and strongly reinforce the NCEP guidelines for the primary prevention of CHD events. ${ }^{2,4,8-10}$

Based on the results of these studies, a new paradigm for preventive cardiology has emerged. This paradigm suggests that patients should be treated based on the magnitude of their cardiovascular risk rather than their demographic characteristics. In clinical practice this means that all high-risk patients, including women and appropriate older patients, should be treated before they develop CHD, the disease most likely to cause death and disability for both men and women in the United States. ${ }^{1,2}$

Evidence-based medicine requires a comprehensive evaluation of the literature, using generally accepted overview or meta-analysis techniques. ${ }^{11}$ Rigorous reviews of the effects of lipid-lowering therapy on CHD morbidity and mortality rates have established the effectiveness and safety of treating high-risk persons to prevent cardiac events. ${ }^{3,12,13}$ The Froom et al article is an opinion piece that is selective in its use of the medical literature. It does not use a standard review methodology and neglects several important primary prevention trials, including trials with substantial numbers of female participants. ${ }^{3,8,14}$ Based on all of the available clinical trial data, the most recent estimates of the benefits of cholesterol reduction indicate a 37 percent reduction in the risk of cardiac deaths $(R R=0.63,95$ percent confidence interval [CT $0.45-0.89$ ) and a 26 percent reduction in overall mortality $(\mathrm{RR}=0.74,95$ percent $\mathrm{CI}=0.58$ - 
0.95 ) by statins, without important increases in noncardiovascular diseases or mortality, including cancer. ${ }^{3}$ A recent review of lipid-lowering therapy for primary prevention indicated that a 10 percent reduction in total cholesterol levels resulted in a 25 percent reduction in $\mathrm{CHD}$ events within 5 years (95 percent CI $15-35$ percent)..$^{13}$

To illustrate this point, it is useful to evaluate the landmark primary prevention trial that used one of the more potent lipid-lowering agents (pravastatin). WOSCOPS 4 enrolled 6595 men between the ages of 45 and 64 years, without antecedent cardiovascular events, but with total cholesterol levels greater than $252 \mathrm{mg} / \mathrm{dL}$. Those who had a low-density lipoprotein (LDL) cholesterol level greater than $155 \mathrm{mg} / \mathrm{dL}$ after 4 weeks of dietary therapy were randomized to receive either placebo or pravastatin ( $40 \mathrm{mg}$ daily at bedtime) and were observed for 5 years. The primary endpoint of nonfatal MI or CHD death was reduced by 31 percent ( 95 percent CI $17-43$ percent, $P<0.001$ ). Similar reductions in death rates from all cardiovascular causes ( 32 percent, $P=0.033$ ) and the need for coronary revascularization procedures $(37$ percent, $P=0.009$ ) were observed. All-cause mortality was reduced by 22 percent ( 95 percent $\mathrm{CI}$ $0-40$ percent, $P=0.051$ ).

Froom et al emphasize that the $P$ value for allcause mortality in WOSCOPS (0.051) did not cross the traditional threshold for statistical significance $(0.05)$ and state that all-cause mortality was unaffected. ${ }^{7}$ This conclusion misinterprets and artificially constrains the meaning of $P$ values reported in clinical trials. A more accurate interpretation of the mortality reduction observed in WOSCOPS reflects the 95 percent confidence limits surrounding the 22 percent risk reduction observed in this study ( 95 percent CI $0-40$ percent). ${ }^{4}$ WOSCOPS was able to show, with 94.9 percent certainty, that pravastatin either did not affect all-cause mortality or reduced it by up to 40 percent. In clinical terms, at a minimum, pravastatin therapy was safe and did not increase mortality; most likely it reduced all-cause mortality.

By multivariate analysis and adjustment for baseline variables, the mortality reduction observed in WOSCOPS was 24 percent, with a statistically significant $P$ value (0.037) $1{ }^{15}$ Analysis of the WOSCOPS patients who took at least three fourths of the prescribed medication indicated that pravastatin reduced all-cause mortality by 32 per- cent $(P=0.015) .{ }^{16}$ To evaluate WOSCOPS primarily on all-cause mortality, which was not the primary endpoint of the trial, ignores the dramatic reduction in morbidity that was associated with the undeniable benefits of pravastatin in regard to preventing nonfatal MIs, CHD death, and coronary revascularization procedures in a relatively lowrisk population (placebo event rate 9.3 percent). ${ }^{4}$

Although reluctant to ascribe any mortalityreducing benefit to cholesterol therapy in WOSCOPS because of a 0.001 variance from 0.05 , Froom et al feel comfortable criticizing the Helsinki Heart Study and the Lipid Research Clinics Coronary Primary Prevention Trial for "nonsignificant increases" in noncardiac illnesses. Such selective use of evidence is statistically inappropriate and very misleading.

The benefits of primary prevention in WOSCOPS and other trials compare favorably with those of other common, highly effective primary and secondary preventive therapies such as aspirin, $\beta$-blockers, lipid-lowering therapy, and estrogen replacement therapy (Table 1)..$^{4-6,17-22}$ In a follow-up report from the WOSCOPS investigators, the benefit of pravastatin therapy for primary prevention of $\mathrm{CHD}$ events was compared with the benefit obtained by lipid-lowering for secondary prevention of CHD events and blood pressurelowering therapy for stroke prevention in mildly to moderately hypertensive middle-aged men in the Medical Research Council trial. ${ }^{14,18}$ After 5 years of therapy, two to four times fewer middleaged men with hypercholesterolemia needed to be treated with pravastatin to prevent one first $\mathrm{CHD}$ event than needed to be treated with a $\beta$-blocker or diuretic to prevent one stroke.

From a clinical practice standpoint, two important issues remain: (1) whether the magnitude of the benefits obtained by lipid-lowering therapy are clinically relevant given the relatively low placebo event rates in the primary prevention trials, and (2) whether similar benefits could be expected in subgroups of patients who would not have qualified for these studies (ie, nonwhites, women, older adults). The answers to these questions have important implications for health care policy, because the screening and treatment of all hypercholesterolemic patients might not be desirable or economically feasible.

The discussion of hyperlipidemia and CHD events in women and older patients by Froom et al 
Table 1. Numbers Needed to Treat (NNT) to Prevent One Adverse Medical Outcome.

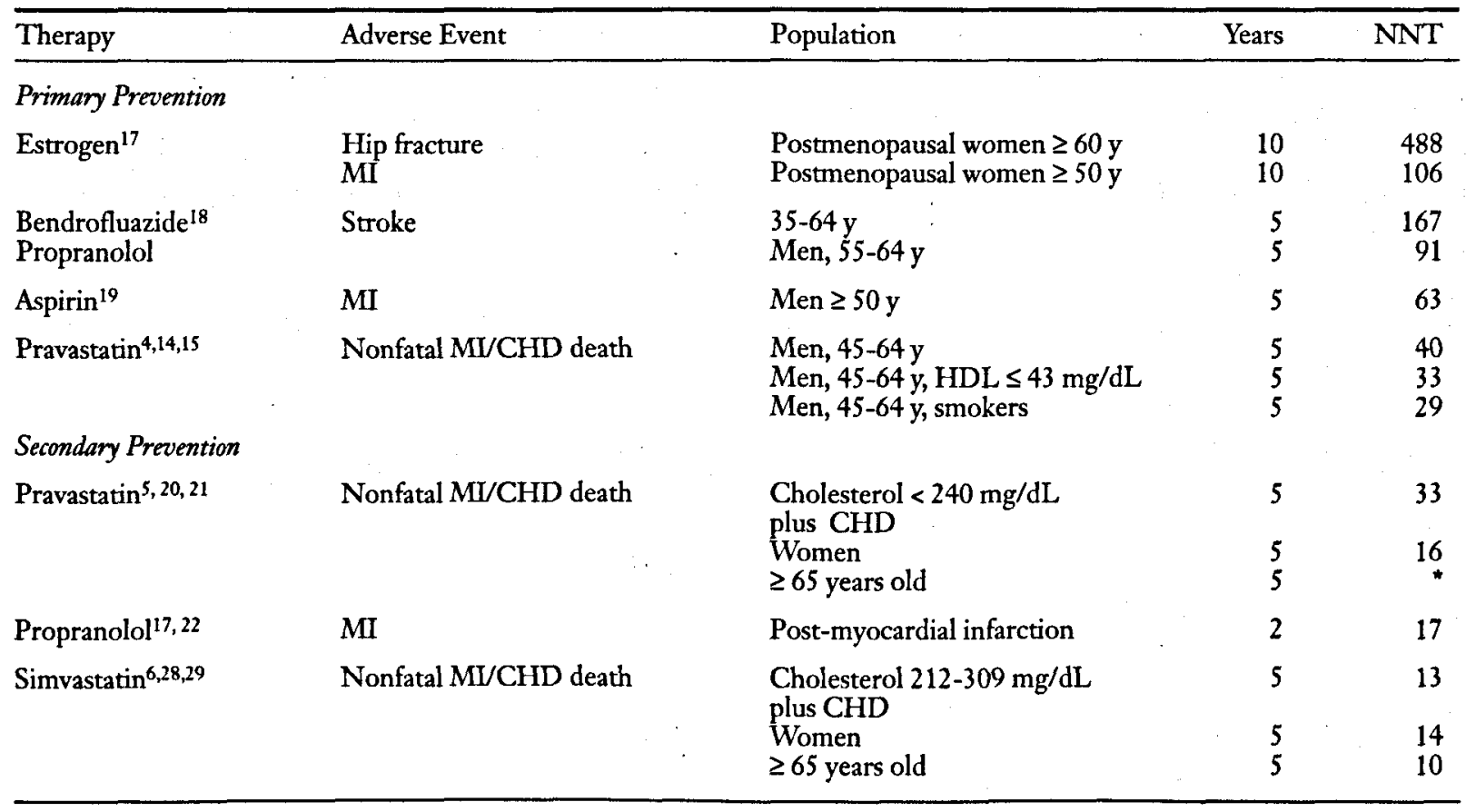

NNT - number needed to treat to prevent one clinical event during the time period specified, MI - myocardial infarction, CHD - coronary heart disease, HDL - high-density lipoprotein.

$32 \%$ relative risk reduction.

is limited to selected observational studies rather than a comprehensive or systematic literature review, and their conclusions are not based on the results of randomized clinical trials.

The main study findings Froom et al use to argue that cholesterol levels do not predict CHD in the elderly actually were based on a small sample (less than 35 percent) from a larger study of older patients. 23,24 This small, incomplete study had several serious limitations, including the use of nonfasting cholesterol values and failure to account for regression-dilution bias, which could have obscured the association between cholesterol levels and clinical endpoints. ${ }^{23}$ More important, the study included only 63 men (less than 16 percent) who had total cholesterol levels exceeding $240 \mathrm{mg} / \mathrm{dL}$, so meaningful statements about hypercholesterolemia in older patients could not be made. ${ }^{23}$ When all of the centers from the trial were evaluated and established risk factors for CHD and markers of poor health were adjusted for, elevated total cholesterol levels strongly predicted the risk of death from $\mathrm{CHD} .{ }^{24}$ In addition, the risk of death from CHD increased in parallel to total cholesterol levels. ${ }^{24}$ The apparent adverse health effects associated with low cholesterol levels were secondary to comorbidity and frailty, which suggests that chronic illness caused low cholesterol levels and increased mortality, not the other way around. 24,25

The safety of statin medications in older patients and women is well-established. 8,20,21,26-29 Indeed, the major lipid-lowering trials that included older patients and women showed clinical benefit equal to or exceeding the benefits obtained in younger, male patients. $8,20,21,26-30$ To deny the benefits of lipid-lowering therapy to dyslipidemic women, women with other risk factors for coronary artery disease, and older patients or to wait until they have a CHD event to begin treatment is to deny a potentially lifesaving therapy for highrisk patients. The new paradigm suggests that the decision to initiate lipid-lowering therapy depends on a patient's risk of experiencing a cardiovascular event, not merely their age, sex, cholesterol level, medical history, or family history as isolated risk factors. This approach strongly supports the NCEP guidelines, which are based on overall risk. ${ }^{2}$

The rationale for the NCEP recommendation to screen for dyslipidemias in young men, premenopausal women, and children from high-risk families is to detect an increased risk of CHD. Detecting increased risk in patients allows them to be counseled and to make informed decisions about 
modifying life habits or receiving treatment before atherosclerotic vascular disease develops. ${ }^{2,31}$ This positive attitude toward the procurement and disclosure of prognostic medical information is diametrically opposed to unsubstantiated concerns of Froom et al about stigmatization and labeling. ${ }^{7}$ Adverse effects of being labeled as a result of knowing about high cholesterol levels have not been shown, but a serious failure of physicians to treat high-risk persons has been shown repeatedly. ${ }^{31-33}$

Determining early which patients are at high risk also facilitates early intervention in the form of nutritional change, physical exercise, weight control, and health education. Coronary atherosclerosis begins in childhood, and it has been established that the total cholesterol level in the third decade of life predicts the rate of development of CHD during the following 30 to 40 years. $^{30,34,35}$ It also is important to ascertain which children and families are at high risk to find out whether they have common genetic cholesterol disorders that are associated with premature CHD. ${ }^{34}$ It is erroneous to assume that the risk associated with atherosclerosis which has taken decades to develop can be mitigated later in life by initiating therapy after a CHD event or a certain age threshold is reached. CHD event rates in patients receiving clearly effective secondary prevention therapies still remain unacceptably high.,6 Waiting for the greater than 30 percent mortality rate associated with the first manifestation of CHD is simply unacceptable.

The cost effectiveness of preventive strategies is a complex issue that requires rigorous methodology, not the type of crude pencil-and-paper estimates presented by Froom et al, which only focused on retail medication and screening costs and did not determine actual costs related to clinical outcomes. In a more sophisticated analysis, the undiscounted cost of pravastatin in the WOSCOPS primary prevention trial was approximately $\$ 12,000$ per year of life saved (PYOLS). ${ }^{36}$ This cost compares quite favorably with other accepted public health interventions, such as medical therapy for severely hypertensive patients (\$20,000 PYOLS), the difference between tissue plasminogen activator and streptokinase for treatment of acute MI $(\$ 22,000$ PYOLS), and hemodialysis for chronic renal failure $(\$ 35,000 \mathrm{PY}$ OLS). A high-cost therapy might be acceptable if a therapy is highly effective. The cost effectiveness of lipid-lowering therapy for high-risk persons has been established firmly. ${ }^{37}$

The most effective strategies for disease prevention include population-based approaches that are low cost and low risk (eg, dietary modification, exercise), and selective targeting of high-risk individuals. ${ }^{2,37,38}$ Dietary and lifestyle modifications effectively reduce cholesterol levels and prevent CHD. ${ }^{2,38-41}$ As Americans have reduced their intake of saturated fats, total cholesterol levels steadily dropped from a mean level of $214 \mathrm{mg} / \mathrm{dL}$ to $203 \mathrm{mg} / \mathrm{dL}$ in approximately one decade. ${ }^{41} \mathrm{~A}$ population-based change from the current American diet to the NCEP nutritional recommendations would be expected to reduce average serum LDL cholesterol values by an average of 10 percent. ${ }^{31,32,40}$ More intensive therapy lowers LDL cholesterol levels even more dramatically and has been shown to reduce CHD events. ${ }^{40,42}$

In summary, screening high-risk patients for cholesterol disorders and treating these disorders are safe strategies that reduce the incidence of $\mathrm{CHD}$ and its complications with consequent reductions in overall mortality rates. The veracity of this observation is supported by powerful data collected in prospective randomized clinical trials and comprehensive overviews of primary prevention trials. ${ }^{3,4-6,8,9,12}$ The magnitude and cost effectiveness of cholesterol therapy for the primary prevention of CHD events are greater than observed with several other accepted medical therapies. ${ }^{36,37}$

The generalizability of the benefits of primary prevention strategies is firmly rooted in the understanding that we as physicians care for patients with composite levels of cardiovascular risk, not collections of individual risk factors and demographic characteristics. Concerns about overprescription of lipid-lowering medications are not borne out by the literature, where underutilization of preventive strategies has been the rule. ${ }^{31-33}$ When evaluating the safety and efficacy of therapies for the prevention and treatment of common illness, such as CHD, it is crucial that appropriate and rigorous scientific methods are used, because of the far-reaching public health and clinical practice implications of inappropriate conclusions. It is time to move beyond selective, narrow opinions of preventive strategies and on to the new paradigm that, on the basis of a proper 
and circumspect analysis of all of the available data, requires finding patients who are at high risk and providing treatment before they develop CHD and its complications. This not only is good science, it is good medicine.

$$
\begin{aligned}
& \text { James H. Stein, MD } \\
& \text { Patrick E. McBride, MD, MPH } \\
& \text { Madison, Wisc }
\end{aligned}
$$

\section{References}

1. American Heart Association. Heart and stroke facts: 1995 statistical supplement. Dallas: The Association, 1995:1-23.

2. National Cholesterol Education Program. Second Report of the Expert Panel on Detection, Evaluation, and Treatment of High Blood Cholesterol in Adults (Adult Treatment Panel II). Circulation 1994;89: 1333-445.

3. Hebert PR, Gaziano JM, Chan KS, Hennekens CH. Cholesterol lowering with statin drugs, risk of stroke, and total mortality. An overview of randomized trials. JAMA 1997;278:313-21.

4. Shepherd J, Cobbe SM, Ford I, Isles CG, Lorimer AR, MacFarlane PW, et al. Prevention of coronary heart disease with pravastatin in men with hypercholesterolemia. West of Scotland Coronary Prevention Study Group. N Engl J Med 1995;333:1301-7.

5. Sacks FM, Pfeffer MA, Moye LA, Rouleau JL, Rutherford JD, Cole TG, et al. The effect of pravastatin on coronary events after myocardial infarction in patients with average cholesterol levels. Cholesterol and Recurrent Events Trial Investigators. N Engl J Med 1996;335:1001-9.

6. Randomised trial of cholesterol lowering in 4444 patients with coronary heart disease: the Scandinavian Simvastatin Survival Study (4S). Lancet 1994;344: 1383-9.

7. Froom J, Froom P, Benjamin M, Benjamin BJ. Measurement and management of hyperlipidemia for the primary prevention of coronary heart disease. $\mathrm{J} \mathrm{Am}$ Board Fam Practice 1998;11:12-22.

8. Furberg CD, Adams HP Jr, Applegate WB, Byington RP, Espeland MA, Hartwell T, et al. Effect of lovastatin on early carotid atherosclerosis and cardiovascular events. Asymptomatic Carotid Artery Progression Study (ACAPS) Research Group. Circulation 1994;90:1679-87.

9. Salonen R, Nyyssonen K, Porkkala E, Rummukainen J, Belder R, Park JS, et al. Kuopio Atherosclerosis Prevention Study (KAPS). A population-based primary prevention trial of the effects of LDL lowering on atherosclerotic progression in carotid and femoral arteries. Circulation 1995;92:1758-64.

10. Manninen V, Tenkanen L, Koskinen P, Huttunen JK, Mänttäri $M$, Heinonen $\mathrm{OP}$, Frick $M H$. Joint effects of serum triglyceride and LDL cholesterol and HDL cholesterol concentrations on coronary heart disease risk in the Helsinki Heart Study. Implications for treatment. Circulation 1992;85:37-45.
11. Demets DL. Methods for combining randomized controlled clinical trials: strengths and limitations. Stat Med 1987;6:341-50.

12. Holme I. An analysis of randomized trials evaluating the effect of cholesterol reduction on total mortality and coronary heart incidence. Circulation 1990;82: 1916-24.

13. Law MR, Wald NJ, Thompson SG. By how much and how quickly does reduction in severe cholesterol concentrations lower risk of ischemic heart disease? BMJ 1994;308:367-72.

14. West of Scotland Coronary Prevention Study: identification of high-risk groups and comparison with other cardiovascular intervention trials. Lancet 1996; 348:1339-42.

15. Baseline risk factors and their association with outcome in the West of Scotland Coronary Prevention Study. The West of Scotland Coronary Prevention Study Group. Am J Cardiol 1997;79:756-62.

16. Shepherd $J$. The West of Scotland Coronary Prevention Study (WOSCOPS): benefits of pravastatin therapy in compliant subjects. The West of Scotland Coronary Prevention Study Group. Circulation 1996; 94(suppl):I-539. Abstract.

17. Newnham HH, Silberberg J. Coronary heart disease. Women's hearts are hard to break. Lancet 1997;349 (Suppl 1): S13-6.

18. MRC trial of treatment of mild hypertension: principal results. Medical Research Council Working Party. Br Med J Clin Res Ed 1985;291:97-104.

19. Final report on the aspirin component of the ongoing Physicians' Health Study. Steering Committee of the Physicians' Health Study Research Group. N Engl J Med 1989;321:129-35.

20. Lewis SJ, Mitchell J, East C, Glasser SP, Grant J, Johnstone DE, et al. Older patients in CARE benefit from pravastatin post myocardial infarction. CARE Investigators. J Am Coll Cardiol 1997;29:375A.

21. Lewis SJ, Mitchell JS, East C, Glasser SP, Grant J, Johnston DE, et al. Women in CARE have earlier and greater response to pravastatin post myocardial infarction. Circulation 1996;94:1-12.

22. A randomized trial of propranolol in patients with acute myocardial infarction. I. Mortality results. JAMA 1982;247:1707-14.

23. Krumholz HM, Seeman TE, Merrill SS, Mendes de Leon CF, Vaccarino V, Silverman DI, et al. Lack of association between cholesterol and coronary heart disease mortality and morbidity and all-cause mortality in persons older than 70 years. JAMA 1994;272: 1335-40.

24. Corti MC, Guralnik JM, Salive ME, Harris T, Ferrucci L, Glynn RJ, et al. Clarifying the direct relation between total cholesterol levels and death from coronary heart disease in older persons. Ann Intern Med 1997;126:753-60.

25. Iribarren C, Reed DM, Burchfiel CM, Dwyer JH. Serum total cholesterol and mortality. Confounding factors and risk modification in Japanese-American men. JAMA 1995;273:1926-32. 
26. LaRosa JC, Applegate W, Course JR 3rd, Humminghake DB, Grimm R, Kropp R, et al. Cholesterol-lowering in the elderly. Results from cholesterol reduction in seniors program (CRISP) pilot study. Arch Intern Med 1994;154:529-39.

27. Santinga JT, Rosman HS, Rutenfire M, Maciejko JJ, Kobylak L, McGovern ME, Behounek BD. Efficacy and safety of pravastatin in the long-term treatment of elderly patients with hypercholesterolemia. Am J Med 1994;96:509-15.

28. Pedersen TR, Kjekshus J, Pyörälä K, Wilhelmsen L, Haghfeld T, Thorgeirson G. Effect of simvastatin on survival and coronary morbidity in coronary heart disease patients 65 or older. Scandinavian Simvastatin Survival Study (4S) Group. Circulation 1995; 92(suppl):I-672. Abstract.

29. Miettinen TA, Pedersen TR, Pyörälä K, Kjekshus TK. Cholesterol lowering with simvastatin reduced major coronary events, revascularization procedures and cardiovascular hospitalizations in women participating in the Scandinavian Simvastatin Survival Study (4S). Circulation 1996;94:1-12.

30. Kane JP, Malloy MJ, Ports TA, Phillips NR, Diehl JC, Havel RJ. Regression of coronary atherosclerosis during treatment of familial hypercholesterolemia with combined drug regimens. JAMA 1990;264: 3007-12.

31. Cleeman JI, Grundy SM. National Cholesterol Education Program recommendations for cholesterol testing in young adults. A science-based approach. Circulation 1997;95:1646-50.

32. McBride $\mathrm{P}$, Plane $\mathrm{MB}$, Underbakke G, Brown $\mathrm{R}$, Schrott $\mathrm{H}$. Cholesterol management of CVD patients by primary care physicians. Can J Cardiol 1997;13: 29B.

33. Stafford RS, Blumenthal D, Pasternak RC. Variations in the cholesterol management practices of U.S. physicians. J Am Coll Cardiol 1997;29:139-46.

34. American Academy of Pediatrics. National Cholesterol Education Program. Report of the Expert Panel on Blood Cholesterol Levels in Children and Adolescents. Pediatrics 1992;89(3 Pt 2):525-84.

35. McHill HC Jr, McMahan CA, Malcom GT, Oalmann MC, Strong JP. Effects of serum lipoproteins and smoking on atherosclerosis in young men and women. The PDAY Research Group. Pathological Determinants of Atherosclerosis in Youth. Arterioscler Thromb Vasc Biol 1997;17:95-107.

36. Shepherd J. The cost-effectiveness of preventing initial coronary events with pravastatin: results of the West of Scotland Coronary Prevention Study Economic Analysis. West of Scotland Coronary Prevention Study Economic Analysis Group.J Am Coll Cardiol 1997;29:168A.

37. Goldman L, Gordon DJ, Rifkind BM, Hulley SB, Detsky AS, Goodman DW, et al. Cost and health implications of cholesterol lowering. Circulation 1992;85:1960-8.

38. Chait A, Brunzell JD, Denke MA, Eisenberg D, Ernst $\mathrm{ND}$, Franklin FA Jr, et al. Rationale of the diet-heart statement of the American Heart Association. Report of the Nutrition Committee. Circulation 1993;88: 3008-29.

39. Krauss RM, Decklebaum RJ, Ernst N, Fisher E, Howard BV, Knopp RH, et al. Dietary guidelines for healthy American adults. A statement for physicians and health professionals by the Nutrition Committee, American Heart Association. Circulation 1996;94: 1795-800.

40. Denke MA. Cholesterol-lowering diets. A review of the evidence. Arch Intern Med 1995;155:17-26.

41. Johnson CL, Rifkind BM, Sempos CT, Carroll MD, Bachorik PS, Briefel RR, et al. Declining serum total cholesterol levels among US adults. The National Health and Nutrition Examination Surveys. JAMA 1993;269:3002-8.

42. Ornish D, Brown SE, Scherwitz LW, Billings JA, Armstrong WT, Ports TA, et al. Can lifestyle changes reverse coronary heart disease? The Lifestyle Heart Trial. Lancet 1990;336:129-33.

\section{Process Instead of Prayer: Moving Toward Active Management
of Patient Care}

Physicians have traditionally been skeptical of practice guidelines and protocols in the belief that they are too rigid to be relevant to the ever-changing, highly variable world of medicine, in the faith that good, well-trained physicians will do the right thing, at least most of the time. Numerous articles in the recent literature, however, have shown that especially in preventive medicine, where the potent reinforcers of adverse outcomes are delayed and infrequent, faith and good intentions will not get the job done.

Preventive care must be institutionalized. Systems must be developed in each primary care practice to define a process that ensures that high-quality preventive care will be offered to all patients.

Leininger and colleagues, ${ }^{1}$ in a report sponsored by the American Cancer Society, have re-

Submitted 25 September 1997.

From the University of Rochester School of Medicine and Dentistry, Rochester; and Tri-County Family Medicine, Cohocton, NY 14826. Address reprint requests to Paul S. Frame, MD, Tri-County Family Medicine, 25 Park Ave, Cohocton, NY 14826. 